Torrego Egido, L. \& Martínez Scott, S. (2018). Sentido del método de proyectos en una maestra militante en los Movimientos de Renovación Pedagógica. Revista Electrónica Interuniversitaria de Formación del Profesorado, 21(2), 1-12.

\title{
Sentido del método de proyectos en una maestra militante en los Movimientos de Renovación Pedagógica
}

\author{
Luis Torrego Egido y Suyapa Martínez Scott
}

Facultad de Educación de Segovia. Universidad de Valladolid

\section{Resumen}

El trabajo por proyectos en la escuela, ahora más frecuentemente conocido por aprendizaje basado en proyectos (ABP), no ha aparecido en los últimos años ni es una moda inventada por quienes comercializan o especulan con la innovación educativa. Con el nombre de método de proyectos, es una propuesta que cuenta con más de cien años de historia y que aparece vinculada a la crítica de la enseñanza tradicional y a la unión de la educación con la transformación social. Los Movimientos de Renovación Pedagógica (MRP) muestran también ese mismo afán crítico y transformador, tanto de la escuela como de la sociedad injusta en la que viven. En este artículo se realiza, recurriendo a la investigación biográficanarrativa, un estudio del significado del trabajo por proyectos para una maestra con más de treinta años de experiencia en la utilización de esta metodología, con una larga trayectoria de militancia en MRP. Se analizan las relaciones entre el uso de proyectos y la identidad docente conformada en su dilatada experiencia como militante, las características del trabajo por proyectos que considera esenciales y las finalidades educativas y sociales que pretende.

\section{Palabras clave}

Aprendizaje basado en proyectos; Movimientos de Renovación Pedagógica; investigación biográfico-narrativa; educación infantil.

\section{Contacto:}

Luis Torrego Egido, luismariano.torrego@uva.es, Facultad de Educación de Segovia, Campus María Zambrano, Plaza de la Universidad, 1, 40005, Segovia (ESP). 


\title{
Sense of the method of projects in a militant teacher in the Pedagogical Renewal Movements
}

\begin{abstract}
Works by project at school, better known now as project-based learning (PBL) has not appeared in recent years, nor is a fashion invented by those who market or speculate with educational innovation. With the name of method of projects, it is a proposal with a history of more than one hundred years and that appears linked to the traditional education criticism and to the union of education with social transformation. The Pedagogical Renewal Movements (PRM) also show that same critical and transforming desire, at both school and the unjust society in which they live. In this article, with the help of a biographical-narrative research, a study is carried out about the meaning of works by project for a teacher with over thirty years' experience using this methodology and a long history of MRP militancy. The relationships between the use of this methodology and the teaching identity conformed by her extensive experience as a militant, the characteristics of work by projects that she considers essential, and the educational and social purposes she intends, are analyzed.
\end{abstract}

\section{Key words}

Project Based Learning; Movements of Pedagogical Renewal; biographical-narrative research; child education.

\section{Introducción}

No podemos considerar al método de proyectos, ni siquiera en sus orígenes, como una iniciativa pedagógica aislada, pues tiene una clara relación con otras propuestas nacidas en diferentes latitudes. Algún autor (Hernández, 1996; Miñana, 1999) ha visto similitudes muy destacables con la escuela del trabajo de Makarenko en Rusia y con las ideas desarrolladas por Kerchensteiner en Alemania, los centros de interés de Decroly, la escuela popular de Celestin Freinet,... Hoy asistimos a un renovado interés por el método de proyectos, renombrado como aprendizaje basado en proyectos (ABP), pero hay una considerable confusión en torno a los presupuestos de esta metodología, pues se presenta, en ocasiones, como una simple técnica innovadora y propugna la adaptación social del alumnado mediante un aprendizaje más eficaz.

Por eso parece oportuno volver la mirada al significado de esta propuesta. En este trabajo lo hacemos sirviéndonos de la experiencia de una maestra cuya identidad docente se ha forjado en el crisol de los Movimientos de Renovación Pedagógica (MRP). Para buscar el sentido de su experiencia con el trabajo por proyectos resulta necesario, previamente, hacer un bosquejo de las notas esenciales con que fue concebido en sus orígenes el método de proyectos y exponer qué son los MRP y cuál es el contexto en el que surgieron y la esencia de estos órganos de movilización pedagógica.

\section{El método de proyectos}

En enero de 1934 se publica en Madrid, en la revista Escuelas de España, - un medio que impulsó la renovación pedagógica en España, principalmente en el medio rural, que reclama 
hoy la necesidad de un estudio que lo rescate de la niebla del olvido - un artículo del que es autor uno de los tres coeditores de la publicación, el maestro segoviano David Bayón. El artículo se titula "Fundamentos del método de proyectos". En él Bayón utiliza principalmente la teoría de John Dewey para esbozar en qué consiste el método de proyectos y llega a una definición: un proyecto es un acto problemático llevado a completa realización en su ambiente natural. El maestro segoviano sitúa en la base del método de proyectos dos pensamientos de Dewey: el reconocimiento de que las ocupaciones activas (las cosas que hacer) son las propiamente escolares y el asignar el carácter de educativas a las actividades que "puedan servir de tipo" a situaciones sociales.

Pocos años antes, Fernando Sainz (1931), otro maestro renovador, había publicado un libro en el que describe la esencia de este modelo: el método de proyectos es la más exacta interpretación del aprender haciendo. Sainz se pregunta por qué no organizar la escuela utilizando como eje un plan de quehaceres similar al que se desarrolla fuera, en la calle, en la sociedad. Aprender haciendo y la escuela como laboratorio social vuelven a ser citados como los pilares del método de proyectos.

El método de proyectos hunde sus raíces, pues, en los afanes renovadores del movimiento de la educación progresista estadounidense. Precisamente este mismo año se cumple el centenario de la publicación del ensayo de Kilpatrick (1918), en el que describe y delimita el método por primera vez, posibilitando su extensión a todo el mundo. El método de proyectos no es sólo una forma de organizar el proceso de enseñanza-aprendizaje, sino que tiene como finalidad que el alumnado pueda desarrollar la independencia y la responsabilidad y practicar modos de comportamiento social y democrático (Knoll, 1997).

Según Hernández (1996, p. 48-49), para acercarnos a la comprensión de lo que supone el método de proyectos hay que considerar las cuatro condiciones que Dewey atribuye a lo que denomina ocupaciones constructivas (el propio Dewey afirmaba que estas ocupaciones constructivas han ido ganando espacio en el aula escolar y se las conoce como proyectos): es fundamental el interés del alumno o alumna, dirigido a un objetivo; centrarse en la actividad (aunque no todas las actividades valen; Dewey descarta las actividades triviales); el proyecto, en su desarrollo, ha de presentar problemas que despierten nueva curiosidad, el deseo de seguir aprendiendo; para la ejecución del proyecto debe contarse con un considerable margen de tiempo. Si se asegura el cumplimiento de estas condiciones surge el proyecto, no como una sucesión de actos inconexos, sino como una actividad ordenada con coherencia, en la que un paso prepara la necesidad del siguiente y en la que cada uno de ellos se añade a lo que ya se ha hecho y lo trasciende de un modo acumulativo.

Félix Martí Alpera, otro educador que impulsó la renovación, formula otras exigencias del proyecto, que reafirman su carácter inclusivo y profundamente educativo:

Este debe ser esencialmente la obra de los niños y si sólo una insignificante minoría es capaz de trabajar en él, es que ha sido mal elegido. Además, en los proyectos escolares lo importante no es la obra terminada, sino el proceso de su realización; no es la meta, sino el camino (Martí Alpera, 1934, p. 4).

Desde sus inicios, el método de proyectos no ha conocido una única manera de concretarse. El propio Martí Alpera señala que los autores norteamericanos distinguen, ya en ese momento, al menos diecisiete maneras diferentes de interpretar el método de proyectos.

Hoy se vuelve a hablar con mucha frecuencia de proyectos en educación. Aparece, eso sí, con otra denominación: aprendizaje basado en proyectos, o, siendo más exactos, se utilizan, simplemente, las siglas: ABP. 
$Y$ podemos encontrarnos, ahora, cuando se habla de ABP con aproximaciones conceptuales que aún guardan huellas de ese empeño transformador - aunque se centre en presentarlas desde el punto de vista de su eficacia para la adquisición de las competencias básicas -, como la propuesta por Trujillo (2012) y por Pozuelos y Rodríguez (2008, pp. 11-13) para quienes el $A B P$ se puede caracterizar como un proceso de enseñanza basado en el alumnado, en el cual se atienden o se tienen en cuenta sus intereses así como se les involucra o se insta a su implicación; además, el ABP favorece la integración del currículum, el aprendizaje como efecto de un proceso de investigación, el desarrollo profesional del profesorado, la inclusión de la diversidad, el rechazo de la rutina y la monotonía y una perspectiva democrática de la educación.

Sin embargo, otras presentaciones de esta metodología ignoran la potencialidad social y la profundidad transformadora que contiene: el Aprendizaje Basado en Proyectos ( $A B P$ ), una estrategia didáctica, en la que los estudiantes, organizados en grupos, desarrollan proyectos basados en situaciones reales. Se crean grupos de alumnos para investigar proyectos y proponer soluciones, trabajando de esta forma habilidades que necesitarán para su carrera profesional y para su vida: cooperación, investigación, pensamiento creativo, comunicación, gestión del tiempo... (Boss y Krauss, 2007)

En estas presentaciones se especifica la finalidad del ABP: "el objetivo es que el alumno aprenda haciendo, en la acción. Se trata de aprender a afrontar el estilo de trabajo que necesitará en su futura práctica profesional. Lo que se busca es que el estudiante aprenda a aprender". (Imaz, 2015, p. 682)

El impulso renovador, cívico, democrático; la búsqueda de la creación de sujetos activos, críticos y configuradores de la sociedad en la que viven; la formación de seres humanos protagonistas de la historia se sustituye ahora por unos fines más utilitaristas: la preparación para el mundo del trabajo.

Y la creatividad presente en el origen pasa a ser reemplazada por una versión técnica de la metodología, que se reduce a una serie de pasos que hay que dar para que el ABP funcione con eficacia. Es como si fuera un manual de instrucciones. Por si fuera poco, en algunos foros educativos se empieza a comentar que ya existe el ABP realizado mediante libros de texto. Tal y como señala Araguz (2015) muchos docentes "hacen proyectos" en lugar de "trabajar por proyectos" y recomienda, para evitar el desperdicio de esta oportunidad educativa, releer a "los clásicos".

\section{Los Movimientos de Renovación Pedagógica (MRP)}

Para entender qué son los MRP hay que tener en cuenta el contexto social y político en el que nacen y crecen; así lo afirma Martínez Bonafé:

Nuestros Movimientos de Renovación Pedagógica son la concreción de un particular devenir histórico, y la forma concreta que en el espacio de la escuela toman las luchas emancipatorias - sociales y profesionales - del sector más vanguardista de los trabajadores de la enseñanza (Martínez Bonafé, 1989, p. 12).

Los MRP configuran, tal y como señala Hernández Díaz, un movimiento amplio, complejo y muy diversificado conformado por el profesorado al final de la Dictadura y los inicios de la denominada transición y con persistencia más o menos activa hasta nuestros días. En sus orígenes están algunas experiencias históricas emblemáticas y varios pensadores con un discurso educativo crítico:

...las propuestas del movimiento de la "Escuela Nueva"; la experiencia de la "Escuela Moderna" de Ferrer y Guardia y la ILE; las aportaciones de grandes 
educadores como Freinet, Milani, Freire o Neill entre otros; o las contribuciones teóricas realizadas por la sociología crítica de la educación (Pericacho, 2015, p. 130).

Así pues, los MRP renacen clandestinamente en el tardofranquismo para plantear la defensa de una escuela democrática y pública, sensible a la equidad social: "lo que les une a todos ellos es que son asociaciones representativas de una renovación pedagógica inmersa en lo social" (Esteban, 2016, p. 260). Hay una clara voluntad ideológica de renovación educativa, pero también de transformación social, hasta el punto de que este último autor los califica de movimientos sociales, ya que en su voluntad de alternativa a lo establecido, reflejan así las condiciones para la renovación pedagógica en la X Escola d'Estiu de Barcelona de 1975:

Para que una renovación pedagógica pueda darse son necesarias unas condiciones sociopolíticas nuevas, con libertades democráticas. En este marco, el movimiento renovador general será impulsado colectivamente por los enseñantes conjuntamente con todas las demás fuerzas sociales (...) Los movimientos de renovación pedagógica comprometidos en esta tarea, y en este contexto, deberán intervenir en la planificación y programación general de la Nueva Escuela Pública. (MRP, 1975, p. 12)

Una dimensión esencial de los MRP es la formación del profesorado, comenzando por la de sus integrantes. Quizás su recurso más conocido sean las escuelas de verano, convertidas, a la vez, en un espacio de formación del profesorado y de lucha por la democracia, una palanca para articular una nueva sociedad, una nueva política y una nueva escuela (Fernández de Castro y Rogero, 2001). Y esa formación, teñida de compromiso social, procura conseguir una educación renovada, centrada en la actividad del alumnado, sujeto de su propio proceso de formación, globalizada e interdisciplinar, propiciadora del desarrollo integral del alumnado (Martínez Bonafé, 1998, p. 188). Por eso ha de basarse en la pedagogía activa.

Como señala Pinto (2016), los MRP propugnan una autoformación colectiva, buscando y organizando la cooperación entre profesionales educativos, por eso establecen "plataformas autónomas de debate, contraste de experiencias e investigación, tanto en el seno de los MRP como en el conjunto de colectivos y grupos implicados en la tarea educativa" (Llorente Cortés, 2003, p.72).

Esta autoformación incide en la práctica de sus integrantes, pero va mucho más allá, pues inciden de manera decisiva en la formación de la identidad docente:

Los MRP protegen de la soledad y de los errores; defienden de las medidas autoritarias y los prejuicios; dan cobijo ideológico y comprensión; facilitan experiencias educativas y personales; enseñan a compartir y a escuchar; se crean como sujeto colectivo; dan ilusiones de que otra escuela es posible, y fuerzas para continuar andando (Pinto, 2016, p. 354).

\section{Metodología}

Este estudio se sirve del método biográfico-narrativo. En realidad, compartimos con Bolivar y Domingo (2006) que no estamos ante una metodología de recogida y análisis de datos, sino que nos situamos en un enfoque que pretende la exploración de los significados profundos de las historias de vida y compartimos los postulados básicos por ellos definidos. Como señalan estos autores, las percepciones de la práctica y el conocimiento experiencial son difícilmente transmisibles de otro modo que no sea el narrativo y el constructivista, 
entendido este como la atribución de significados a las múltiples historias que se van reconstruyendo en torno a pasajes o episodios que se refocalizan en función del punto de interés y de la comprensión actual. A ello hemos de añadir el carácter contextual del enfoque: las narraciones encuentran sentido dentro de los contextos en que son contadas y en los que se produjeron.

Dentro de la clasificación de las historias de vida realizada por McKernan (1999), se encuadra dentro de las temáticas, pues la investigación se centra en el tema del trabajo por proyectos y se profundiza en él. Para la elaboración de la misma, se han seguido las etapas enunciadas por Cornejo, Rojas y Mendoza (2008): el momento preliminar, con la elección del tema y el ángulo de abordaje del mismo; los contactos y negociaciones, marcados por la información a la protagonista de los objetivos y el contenido de la investigación y por el consentimiento informado, la garantía del anonimato y la libertad para interrumpir su colaboración; la recolección de los datos mediante las entrevistas (incluida la transcripción de las mismas); el análisis de los relatos, tras la devolución de las transcripciones a la maestra y la escucha de los comentarios e interpretaciones realizados por ella.

Este trabajo, pues, se basa en una historia de vida de una maestra que se ha realizado mediante siete entrevistas llevadas a cabo en diferentes lugares: el domicilio de la maestra, la escuela en la que trabaja, una sala de las instalaciones en las que se realizaba un Encuentro de MRP y en un viaje en tren de larga distancia. Entre la primera entrevista, realizada en septiembre de 2017, y la última han transcurrido casi cuatro meses. Las entrevistas han tenido una duración que varía entre los poco más de treinta minutos y la hora y veinticinco minutos.

Las entrevistas se han completado con fotografías aportadas por la maestra, en las que se puede ver el desarrollo de sus clases, con el alumnado de las mismas en acción. No todas corresponden al trabajo por proyectos, sino que se incluyen otras actividades dentro y fuera del aula. También se han observado producciones escolares resultantes del trabajo realizado por los niños y niñas.

El análisis, como ya se ha dejado entrever, se ha realizado contando con el criterio de la protagonista y se ha guiado por dos procedimientos descritos por Mancila, Soler, Moyano, y Calvo (2012): la búsqueda de significados conjuntamente con la narradora y, tras establecer un análisis temático de dichos significados organizados en las categorías que se exponen en el análisis de resultados, la negociación de la identidad con la protagonista, de modo que se conforme un único análisis reconocido como propio por ambas personas cohistoriadoras. Por eso se expone el relato en lenguaje directo.

\section{Presentando a la maestra}

Como afirma Pujadas (1992) el investigador debe asegurarse que el informante responda a un perfil característico y representativo del universo socio-cultural que va a estudiar. Por ello, la protagonista de esta investigación es una maestra que ha desarrollado su docencia 38 cursos- mayoritariamente en educación infantil, en Catalunya. Conoció en sus primeros años de ejercicio profesional, por medio de una compañera, de la existencia de los MRP y comenzó una participación muy activa en las actividades y en la organización de estos movimientos, como una forma de manifestar su compromiso educativo. Esta implicación se mantiene hasta hoy, cuando ejerce en una escuela de Castilla, y reconoce que la ha influido de modo determinante tanto en su labor como maestra como en su faceta de directora de su escuela, de formadora de otros docentes y como persona que ama la educación. 


\section{Análisis de resultados}

\section{Qué es el trabajo por proyectos. Los comienzos}

No me acuerdo exactamente del tiempo en que llevo trabajando por proyectos, pero hace más de treinta años ya había empezado a trabajar con una metodología más parecida a un centro de interés que al aprendizaje por proyectos. Empecé con los MRP y en este entorno empecé a trabajar por proyectos, así es que he conocido a mucha gente que los ha puesto en práctica.

No quiero definir el aprendizaje por proyectos, pero puedo decir que es el método más natural de aprender. Se trata de potenciar la curiosidad en los niños y en las niñas y, a partir de ella, ir dando herramientas para resolver esa curiosidad. Esto nos pasa a las personas en todas las facetas de la vida, pero la escuela añade a los proyectos el elemento colectivo, que es muy importante, y la perseverancia y el rigor, que no son cosas que vengan con la especie humana; la especie humana tiende a quedarse en el sofá.

Los niños y niñas aprenden a emocionarse sobre el propio aprendizaje; se emocionan cuando aprenden una cosa y eso realimenta la curiosidad: lo que aprendes te invita a aprender más porque no es una cosa impuesta, sino que es algo sobre lo que tú te has preguntado. Aprenden también estrategias para aprender y a tener en cuenta no sólo tus propios intereses, sino también los de tus compañeros y compañeras, de ahí la importancia de la escuela. A veces aprenden a desaprender todo lo que es la academia, lo escolástico. Es preciso que desaprendan la obediencia per se, el intelectualismo sin contenido. Hay una fase en que cualquier alumno o alumna que haya estado escolarizado en una escuela tradicional tiene que desaprender, si no es así no puede trabajar por proyectos. También el profesorado que quiera trabajar por proyectos ha de desaprender.

Realicé un curso hace muchos años que para mí fue impactante: descubrí que estaba haciendo centros de interés y no trabajo por proyectos. A mí me influyeron también mucho algunos viajes pedagógicos que realicé en mis inicios profesionales. Me impactaron Reggio Emilia, la escuela Decroly, las escuelas freinetianas y las visitas a escuelas que seguían los postulados de Vygotsky. Éramos muy jóvenes y teníamos ganas de cambiar una escuela pública en un pueblo en el que era subsidiaria con respecto a la privada. El contacto con el IMIPAE de Barcelona me acercó a Piaget y a la pedagogía operatoria. También me han influido mucho las lecturas que he realizado, de autores como Freinet, Dewey, Decroly, Makarenko, Freire... Los MRP me pusieron en contacto con los clásicos.

Diferencio entre los centros de interés y el aprendizaje por proyectos, porque los primeros tienen una programación previa en torno a un tema, mientras que los proyectos tienen más carácter de acompañamiento, los protagonistas son los niños y niñas. En el trabajo por proyectos, a diferencia de los centros de interés, no se puede planificar todo previamente. Los proyectos son mucho más globalizadores que los centros de interés y subrayan el trabajo colectivo; esta dimensión colectiva es central. Puede añadirse otra característica muy importante en el trabajo por proyectos: su carácter inclusivo. En el trabajo por proyectos cabe todo el mundo.

Para mí el arranque del proyecto es la incógnita, la pregunta, el querer saber algo. Es lo que pone en marcha todo el proceso. El libro de texto y el proyecto son incompatibles. Bueno, el libro de texto es incompatible con el aprendizaje natural; es un corsé.

Mi último proyecto ha sido en torno al respeto. Los primeros días en esa clase de 4 años costaban un poco: eran niños y niñas muy egocéntricos, con interacciones complicadas, así que partimos de la elaboración de reglas; la primera propuesta la hice yo: en la clase debe haber respeto. Les llamó tanto la atención esta palabra que empezaron a buscar lo que 
quería decir, las formas de respetar. Decidimos hacer un mural para que todo el mundo viera lo que era el respeto, buscamos símbolos que definieran el respeto y fíjate qué curioso que el símbolo que surgió fue el corazón; esto es una cosa preciosa que a mí me emocionó porque ligaban el respeto al amor. Hemos visto que en todas las partes del mundo hay respeto y también hemos hablado del respeto a la naturaleza; todo esto ha ido surgiendo de forma natural, de modo que lo preguntaban ellas. Es una maravilla lo que ha pasado con el proyecto y esto es lo mejor que te puede pasar: que el proyecto te sorprenda a ti.

Los proyectos, incluso, no tienen por qué acabar. Mi último proyecto del curso pasado, sobre las mariposas, no acabó, quedó abierto; trabajamos sobre las mariposas y tuvimos la inmensa fortuna de que de sus huevos nacieran orugas al final de curso, de modo que animamos a las familias a que niños y niñas siguieran aprendiendo durante el verano y así lo hicieron.

Creo que los proyectos tienen que ser documentados. Con esto me refiero a que el proceso de aprendizaje tiene que quedar reflejado en un producto y eso lo pueden realizar los propios niños y niñas, mientras mantienen esa actitud científica que se genera en los proyectos.

Por otra parte, es muy importante el carácter social del proyecto, no sólo por la apertura de la escuela y la implicación de otras personas, por el trabajo en equipo de niños y niñas; me refiero ahora a que el proyecto tiene que conducir al desarrollo de conductas éticas en el alumnado, a la solidaridad con otras personas, al cuidado y respeto del medio ambiente. Los niños y niñas también aprenden el compromiso cuando llevan a cabo el proyecto. Hay otra cosa importantísima en los proyectos: la educación crítica, el desarrollo del espíritu crítico, del pensamiento crítico que dirige su mirada a cuestiones sociales.

\section{La reacción de los demás. El acompañamiento}

Cuando se dice: vamos a trabajar por proyectos y alguien reacciona diciendo: las familias no lo entenderán, estoy ante una cosa que no comprendo. Yo creo que cuando el profesorado está convencido y entiende qué y por qué lo va a hacer, las familias lo entienden. Todo el mundo sabe que una escuela rutinaria, intelectualista, no va a ningún sitio. En cualquier caso, nosotros siempre hemos dado mucha información y hemos ido avanzando con tranquilidad, siempre ha sido una cosa muy compartida: el camino no lo hizo el claustro solo, sino también las familias y el alumnado. En el aprendizaje por proyectos las familias pasan a ser agentes activos en la educación: como los niños y niñas se emocionan, las familias se emocionan. Los niños y niñas se emocionan ante el descubrimiento de una cosa y aumentan su autoestima.

Los proyectos son una forma de abrir la escuela. Aquí donde trabajo ahora -una ciudad- es más difícil, pero en mi destino anterior todo el pueblo estaba al tanto de lo que estábamos trabajando y mucha gente participaba en el proceso, no sólo las familias.

El aprendizaje por proyectos no lo he realizado sola, sino trabajando con otros compañeros y compañeras, pero me ha tocado tirar del carro en muchas ocasiones. Claro, he tenido compañeros que no compartían este método de trabajo y que ponían en duda su eficacia, así es que en su momento dije: vamos a valorarlo, lo hacemos sólo hasta segundo. Cuando lo valoramos, vimos que lo establecido, los aspectos básicos del curriculum, lo aprenden igual, pero lo hacen de forma significativa y, además, aprenden otras cosas. Una niña que trabaja por proyectos está alfabetizada funcionalmente, es competente hablando en los términos actuales del curriculum, pues utiliza los aprendizajes básicos para aprender cosas.

Los compañeros y compañeras que más han criticado el aprendizaje por proyectos lo han hecho porque no se sienten seguros ante lo no programado, ante el grado de 
incertidumbre que conlleva un proyecto. A ellos lo que les gusta es que el niño aprenda en primero esto, en segundo esto y lo otro,... pero esto no existe, no todos lo aprenden. Esto es en lo que más se han centrado para oponerse: en la incógnita. Pero yo procuré tranquilizarlos con los resultados y con la alegría que supone a una maestra trabajar de esta manera, aprendiendo.

Sobre la denominación... en realidad, a mí no me gusta poner etiquetas a las cosas: lo que se trata es de aprender a través de los proyectos. Me gusta trabajar con los niños a partir de sus intereses y de las preguntas que van surgiendo en el aula. Ahora parece que hay una moda en torno al ABP. A mí el ABP me suena a la marca de una moto, qué quieres que te diga. A mí no me gusta ni el ABP ni el PP ni ninguna otra moda. Las modas son las que pervierten la idea del proyecto, que es tan simple, tan natural. Es la forma natural de aprender: hay un reto y tratas de responder a él, buscando rigor, creatividad, alegría, colectivo. $Y$ ese reto puede ponerlo la maestra, que no tiene que ir siempre detrás de los niños y niñas.

\section{La influencia de los MRP}

Mi contacto con los MRP fue una casualidad, una magnífica casualidad, porque yo, que tenía una tendencia natural hacia la renovación, que la buscaba, coincidí con una compañera que estaba en los MRP. Yo ya tuve la suerte de haber recibido una formación inicial como maestra que era disidente con respecto al sistema de la universidad y, además, estudié mientras trabajaba con niños y niñas de 0 a 3 años y, entonces, podía aplicar cosas que aprendía.

Había asistido ya a la escola de estiu de Rosa Sensat, en Barcelona, que para mí era un espacio de libertad política, además de renovación pedagógica. Yo estaba de acuerdo con sus planteamientos; no tuvieron que convencerme, me atraía la militancia pedagógica.

Empecé entonces a leer muchos textos pedagógicos y eso fue muy relevante, pues fundamenté lo que pensaba: en los MRP había gente muy leída y que me influenció, dediqué una parte de mi escaso presupuesto a comprar libros (incluso a robar algunos).

Los MRP me dieron una cosa fundamental: la sensibilidad y la posibilidad de divertirme hablando y reflexionando sobre pedagogía. También me aportaron el carácter militante: éramos gente de acción, lo que hablábamos era para hacerlo. Y había una cosa, que vista ahora, era maravillosa: la igualdad con la que discutíamos, no había jerarquía, aunque yo tuviera admiración hacia algunas de esas personas. Yo, una maestra novel, podía hablar de educación con Marta Mata, de tú a tú... Ahora hay militantes mayores y hay pocos jóvenes, pero entonces, para una joven como yo hablar con Marta Mata era una vivencia excepcional, determinante.

Había muchos prácticos en los MRP y algunos teóricos y estos últimos valoraban mucho la opinión de los que estábamos en la práctica de la escuela. Ahora la universidad apenas está, antes la presencia de profesorado universitario era mucho mayor. Había una especie de discriminación positiva hacia las personas nuevas y hacia las que estaban en la práctica.

Los MRP siempre han tenido un pensamiento volcado hacia la acción, por eso han influido no sólo en mi pensamiento, también en mi práctica. No sólo en mí, sino también en las escuelas en las que he estado. Yo ya era activista política y pasé a ser activista políticopedagógica.

Los MRP me han dado apertura de miras, flexibilidad, en mi postura de izquierdas. Había entonces una presencia de varias izquierdas y un afán de buscar puntos comunes. También aprendí a confrontar ideas, a debatir sirviéndome de argumentos, de fundamentos. Los MRP eran, desde sus inicios, un espacio de convivencia y encuentro y eso ha sido 
fundamental para poderse mantener tanto tiempo. Había una cierta rivalidad "teórica" entre distintos territorios del Estado, pero era una rivalidad sana, estimulante.

Los MRP me han condicionado absolutamente como maestra. Eso se ve, por ejemplo, en la mirada crítica que tengo hacia las tendencias pedagógicas innovadoras, hacia las modas de la innovación. Lo importante no es tanto la forma en sí, sino la forma que encierra una finalidad transformadora. Mis compañeros y compañeras de los movimientos siempre me han valorado mucho esa mirada, como también lo han hecho con mi manera amorosa, afectiva, de decir las cosas. Marta Mata me decía que la conservara...

\section{Discusión y conclusiones}

El relato que acabamos de reflejar nos permite vislumbrar, pese a la limitada extensión del mismo, la complejidad del trabajo escolar mediante proyectos. En realidad, tal y como señala Vergara (2016), el aprendizaje por proyectos es un marco desde el que se piensa el aprendizaje del alumnado. La maestra, al igual que el autor citado, remarca la necesidad de que sea un método abierto y flexible y de que se desarrolle de un modo natural y planificado, a la vez.

Frente a concepciones que definen el ABP como una técnica, como una ruta guiada y planificada previamente o como una de las más destacadas "estrategias metodológicas emergentes" (Sánchez, 2012), nuestra protagonista rechaza las modas educativas y nos lleva a una aproximación fundada y elaborada de este enfoque, una concepción que es muy implicadora, creativa y plena de amor por la educación y de compromiso con ella.

La insistencia en basar el proyecto en los intereses de niños y niñas y en ese respeto por su capacidad de aprendizaje coincide con lo que otras investigaciones (García-Valcárcel y Basilotta, 2017) han encontrado sobre la importancia de la motivación del alumnado con respecto a su propio aprendizaje y con el grado de compromiso que desarrollan con el proyecto.

Destaca la importancia otorgada a las dimensiones colectivas y sociales, que coincide también con las encontradas en algunos estudios sobre el ABP en educación infantil (Remacha y Belletich, 2015), en los que se pone en evidencia cuestiones aquí citadas, como la implicación de las familias, el aumento de la autoestima colectiva o la concienciación y el compromiso medioambiental de niños y niñas.

Por otra parte, la influencia de los MRP en la configuración de la identidad docente de la maestra aparece reflejada con nitidez. Los MRP han sido no sólo un marco formativo de nuestra protagonista, sino que han incidido incluso en lo que podría llamarse la formación del carácter, pues incluye elementos afectivos e ideológicos. Además, compañeros y compañeras de MRP han servido no sólo como participantes en un diálogo renovador, sino también como acompañantes en la iniciación y uso de esta propuesta pedagógica que nuestra maestra nunca ha ejercido en soledad.

Este trabajo, humilde en su pretensión y en su estructura metodológica, creemos que sirve para poner de manifiesto la profundidad del significado de la renovación pedagógica. Frente a etiquetas que esconden tras su apariencia una realidad de enseñanza tradicional modificada cuya lógica permanece inalterada, aquí la renovación es profundamente transformadora: sensible con la con la equidad social, la educación inclusiva, el desarrollo integral de las personas, la apertura de la escuela al entorno, la educación cívica,...

Sirve también para poner de manifiesto la necesidad de estudios sobre el proceso de aprendizaje por proyectos, no sólo sobre los resultados obtenidos; sobre el significado y las 
finalidades atribuidas a la renovación o a la llamada innovación educativa y sobre las influencias recibidas por el profesorado en la formación de su identidad docente.

\section{Referencias}

Araguz, A. (2015, 10 de abril). No todo vale en ABP (Aprendizaje Basado en Proyectos) [Post de blog]. Recuperado de http://blog.educalab.es/intef/2015/04/10/no-todo-vale-enabp-aprendizaje-basado-en-proyectos/

Bayón, D. (1934). Fundamentos del método de proyectos. Escuelas de España, segunda época, 1 (1), $19-28$.

Bolívar, A., \& Domingo, J. (2006). La investigación biográfica y narrativa en Iberoamérica: Campos de desarrollo y estado actual. Forum: Qualitative Social Research (7), 4, 1-43.

Boss, S. \& Krauss, J. (2007).Reinventing Project-Based Learning: your field-guide to realword projects in the Digital Age. Washington-EEUU: ISTE

Cornejo, M., Rojas, R.C. \& Mendoza, F. (2008). La investigación con Relatos de Vida: Pistas y opciones del Diseño Metodológico. Psykhe, 17, 29-39.

Esteban, S. (2016). La renovación pedagógica en España: un movimiento social más allá del didactismo. Tendencias Pedagógicas, 27, 259-284.

Fernández de Castro, I., \& Rogero, J. (2001). Escuela pública. Democracia y poder. Madrid: Miño y Dávila.

García-Valcárcel, A., \& Basilotta, V. (2017). Aprendizaje basado en proyectos (ABP): evaluación desde la perspectiva de alumnos de Educación Primaria. Revista de Investigación Educativa, 35(1), 113-131

Hernández, F. (1996). Para comprender mejor la realidad. Cuadernos de Pedagogía, 243, 4853.

Hernández Díaz, J.M. (2011). La Renovación pedagógica en España al final de la transición. El encuentro de los movimientos de renovación pedagógica y el ministro Maravall. Educació i Historia: Revista d' Història de l'Educació, 18, 81-105.

Imaz, J. I. (2015). Aprendizaje Basado en Proyectos en los grados de Pedagogía y Educación Social: “CCómo ha cambiado tu ciudad?”. Revista Complutense de Educación, 26, 3, 679-696

Kilpatrick , W. H. (1918). The Project Method. Teachers College Record, 19(4), 319-335.

Knoll, M. (1997). The project method: Its vocational education origin and international development. Journal of Industrial Teacher Education, 34(3), 59-80.

Llorente Cortés, M. A. (2003). Los Movimientos de Renovación Pedagógica y la lucha contra la mundialización neoliberal. Tabanque: Revista Pedagógica, 17, 71-86.

Mancila, I., Soler, A.; Moyano, A., \& Calvo, P. (2012). Retos en la investigación narrativa: la construcción de significados, voz y re-presentación. En Rivas, J. I., Hernández, F., Sancho, J. M., \& Núñez, C. (Coords.) Historias de vida en educación: Sujeto, Diálogo, Experiencia. (pp. 55-60). Barcelona: Dipòsit Digital UB. http://hdl.handle.net/2445/32345

Martí Alpera, F. (1934). Ensayos del método de proyectos. Madrid: Revista de Pedagogía. 
Martínez Bonafé, J. (1989). Renovación pedagógica y emancipación profesional. Valencia: Servicio de publicaciones de la Universidad de Valencia.

Martínez Bonafé, J. (1998). Trabajar en la escuela: profesorado y reformas en el umbral del siglo XXI. Madrid: Miño y Dávila.

Mckernan, J. (1999). Investigación, acción y curriculum. Madrid: Morata.

Miñana, C. (1999). El método de proyectos. Documento de trabajo. Universidad Nacional de Colombia, Bogotá, Colombia. Recuperado de http://www.humanas.unal.edu.co/red/files/9612/7248/4193/Artculosmetodo_proyectos.pdf

Movimientos de Renovación Pedagógica (1975). Declaración de la X Escola d’Estiu de Barcelona. Por una nueva escuela pública. Cuadernos de Pedagogía. Suplemento 1, 312.

Pericacho, F. J. (2015). Actualidad de la renovación pedagógica en la Comunidad de Madrid: un estudio a través de escuelas emblemáticas. (Tesis doctoral, Universidad Complutense de Madrid). Recuperado de http://eprints.ucm.es/29332/1/T35915.pdf

Pinto, J. M. (2016). Los Movimientos de Renovación Pedagógica de la Comunidad de Madrid. Análisis de su influencia en el desarrollo profesional docente. (Tesis doctoral, Universidad de Valladolid). Recuperado de https://uvadoc.uva.es/bitstream/10324/22228/1/Tesis1218-170208.pdf

Pozuelos, F. J., y Rodríguez, F. de (2008) Trabajando por proyectos en el aula. Aportaciones de una investigación colaborativa. Investigación en la escuela, 66, 5-27.

Pujadas, J.J. (1992). El método biográfico: El uso de las historias de vida en Ciencias Sociales. Madrid: Centro de Investigaciones Sociológicas.

Remacha, A., \& Belletich, O. (2015). El método de aprendizaje basado en proyectos (ABP) en contextos educativos rurales y socialmente desfavorecidos de la educación infantil. Perspectiva Educacional, Formación de Profesores, 54(1), 90-109.

Sainz, F. (1934). El método de proyectos en las escuelas rurales. Madrid: Revista de Pedagogía.

Sánchez, J. M. (2013). Qué dicen los estudios sobre el Aprendizaje Basado en Proyectos.

[Post de blog]. Recuperado de http://www.estuaria.es/wpcontent/uploads/2016/04/estudios_aprendizaje_basado_en_proyectos1.pdf

Trujillo, F. (2012). Enseñanza basada en proyectos: una propuesta eficaz para el aprendizaje y el desarrollo de las competencias básicas. Eufonía - Didáctica de la Educación Musical, 55, 7-15.

Vergara, J. (2016). Aprendo porque quiero: el Aprendizaje Basado en Proyectos (ABP) paso a paso. Madrid: SM. 